\title{
A Novel Exercise Initiative for Seniors to Improve Balance and Physical Function
}

Sales, Myrla ${ }^{1}$, Polman, Remco ${ }^{2}$, Hill, Keith D ${ }^{3}$, Levinger, Pazit ${ }^{1}$

${ }^{1}$ Sales, Myrla, PhD Candidate, Institute of Sport, Exercise \& Active Living (ISEAL), College of Sport and Exercise Science, Victoria University, Melbourne, Victoria, Australia

${ }^{1}$ Levinger, Pazit, Associate Professor and Group Discipline Leader Exercise Science and Clinical Rehabilitation, Institute of Sport, Exercise \& Active Living (ISEAL), College of Sport and Exercise Science, Victoria University, Melbourne, Victoria, Australia

${ }^{2}$ Polman, Remco, Professor and Head of Department of Psychology, Bournemouth University, Fern Barrow, Poole, United Kingdom

${ }^{3}$ Hill, Keith, Professor and Head of School of Physiotherapy and Exercise Science, Curtin University, Perth, Western Australia, Australia

Corresponding author:

Associate Professor Pazit Levinger

Institute of Sport, Exercise \& Active Living (ISEAL), College of Sport and Exercise Science

Victoria University, PO Box 14428, Melbourne VIC 8001

Email: Pazit.levinger@vu.edu.au T: +61399195525 F: +61399199480

Running Headline:

A Novel Exercise Initiative for Older Adults 
Objectives: To investigate the feasibility, effectiveness and short term effects of an exercise intervention using a novel exercise park in improving senior's balance, physical function and quality of life. Methods: Randomised controlled trial with pre and post intervention design (Baseline and 18-week intervention). Outcomes measures included measures of balance, strength and function as well as quality of life and fear of falling. Multivariate analysis of covariance was used to assess differences between groups (Control and Exercise Intervention) over time. Results: Intervention group showed significant improvement on single leg stance $(\mathrm{p}=0.02$, 95\%CI -8.35 to -.549$)$, knee strength ( $\mathrm{p}<0.01,-29.14$ to -5.86 ), two-minute walk ( $\mathrm{p}=0.02,-19.13$ to -.859$)$ and timed sit to stand ( $\mathrm{p}=0.03,-2.26$ to -.143$)$ tests. Discussion: The exercise park program improved physical function and had high adherence and participation rate. Such intervention has been shown to be safe and therefore might enhance participation in exercise programs for older adults.

Keywords: Balance, Physical Function, Exercise, Exercise Adherence, Exercise Uptake. 


\section{Introduction}

Preventing falls, improving muscle strength, balance and physical function among older adults are key public health priorities. Falls are significantly associated with reduction in quality of life of older adults as well as functional decline (Granacher, Muehlbauer, Gollhofer, Kressig, \& Zahner, 2011), with 30-35\% of older people living in the community and aged over 65 years falling at least once a year (Granacher et al., 2011). After having a fall or being fearful of falling, older people tend to develop depression, anxiety, reduce social contact, decrease activity and mobility, increase use of medications and lose independence and autonomy in their lives (Yardley, Donovan-Hall, Francis, \& Todd, 2006). These factors may also be responsible for affecting daily functioning in older adults by promoting further reductions in muscle strength, balance and gait speed (Van Kan et al., 2009)

Randomized controlled trials focusing on reducing the risk factors of falls, falls prevention and improvement of muscle strength, balance and mobility in older adults have shown that exercise interventions slow down functional losses expected with increased age (Paterson, Jones, \& Rice, 2007). Consequently, exercise interventions are able to improve quality of life and maintain functional independence in older adults (Paterson et al., 2007). However, participation in these exercise or falls prevention interventions are rather low (Yardley et al., 2006), suggesting that older people may be reluctant to participate, or do not feel that interventions are sufficiently appealing or beneficial for them to take part in.

In order to increase exercise uptake and adherence for older adults in a community setting, a unique purpose-built outdoor exercise park was designed to provide a fun but still physically challenging environment for older adults (https://www.youtube.com/watch?v=106jz_w5vcg\&feature=youtube). With this novel concept, 
older adults might feel more inclined to exercise and to adopt a healthier lifestyle given that these playful and purposeful activities are also functional and practical to what they do in their daily living activities (McMahon, Talley, \& Wyman, 2011). Furthermore, older adults tend to partake in initiatives that can help them to maintain independence, autonomy and confidence, and, consequently promoting a more positive self-identity (Evron, Schultz-Larsen, \& Fristrup, 2009). The social interaction and support associated with this type of exercise park could make the sessions more enjoyable given that older adults show preference to exercise in groups (Yardley et al., 2008). Moreover, exercising outdoors has been shown to contribute to significant improvements in mood, self-esteem and reduce levels of depression among older adults (Barton, Griffin, \& Pretty, 2012). Therefore, the aims of this study were to investigate the feasibility, and short-term effectiveness of an exercise intervention using a novel outdoor exercise park designed for seniors in improving their balance, physical function and quality of life.

\section{Methods and Design}

The full description of this study's methods, design, randomization process, exercises and tests performed can be found on the full trial protocol previously published (Sales, Polman, Hill, Karaharju-Huisman, \& Levinger, 2015).

Design

This study was a parallel randomised controlled trial with pre and post intervention design (outcome assessments at baseline and at 18 weeks after participation commencement, and number of falls measured over a 12-month period from enrolment in this study) comparing two groups: an exercise park intervention program for older people and a control group.

\section{Participants}


One hundred twenty community-dwelling people aged between 60 and 90 years old were sought via community health promotion events and advertisement in local newspapers, magazines and online social networking media. Participants were also from diverse settings such as local senior organizations, retirement villages, community centres, senior clubs and associations in Melbourne.

\section{Inclusion and Exclusion Criteria}

Older adults who had one or more falls in the previous 12 months or who were concerned about having a fall were recruited for this study. Volunteers who were generally active and independent in the community with no more than a single point stick used for regular outdoors walking (at least three times per week) were included. More details about inclusion and exclusion criteria are detailed on the study protocol (Sales et al., 2015).

\section{Randomization}

Participants were randomly allocated to one of the following groups: (1) Exercise Park Intervention Group (EPIG) or (2) Control Group (CG). Assessors and participants were not blind to their respective group allocation (EPIG or CG). Block randomization stratification by gender using opaque envelopes was undertaken, so that blocks of 12 participants (6 for intervention group and 6 for control group) were randomized at a time. To accommodate couples (e.g. partners/married couples) participation, randomisation by couple also took place.

Participants from the EPIG underwent an 18-week exercise intervention. The exercise sessions were provided two times a week (each class approximately 1 to 1.5 hours duration) and were supervised by an accredited exercise physiologist. Each session consisted of 5-10 minutes warm-up exercises, followed by 45-75 minutes on the equipment stations, and concluded with 510 minutes of cool down exercises. The exercise classes contained a maximum of 6 participants 
and were circuit-based with the warm up and cool down exercises being performed in a group and the core session being carried out in training pairs. Participants performed exercises that focus on strength, balance, coordination, mobility and flexibility (as detailed on this study protocol (Sales et al., 2015)). Exercisers were paired in stations and an exercise session could include up to eight stations (Table 1). The intervention program was carried out with no cost to the participants.

Participants in the CG were advised to continue with their usual daily activities and met the research team every two weeks to take part in some social activities (nine meetings of twohour duration over 18 weeks of participation).

\section{Treatment/Group Preference}

Each participant's group preference was documented (i.e. as control group, exercise intervention group or no preference) to identify if differences exist between those who received their preferred allocation and those who did not.

\section{Exercise Park}

The senior exercise park used in this project was provided in-kind by Lappset (Lappset Group Ltd., Rovaniemi, Finland) (Figure 2) and was installed at St Bernadette's Community Respite House in Sunshine North, Victoria, Australia. The exercise park consists of a number of components and stations that aim to work on the following aspects of physical performance: Upper body mobility and fine motor skills, balance and coordination, lower limb and upper limb strength, stretching and flexibility.

\section{Study Protocol}

All participants were fully informed about the nature of the study and signed a consent form. This study was approved by the Human Research Ethics Committee of Victoria 
University, Melbourne (Application ID. HRE13-215). The study was designed according to the Consolidated Standard of Reporting Trials (CONSORT) guidelines and publications associated with the trial were reported according the CONSORT 2010 Statement (Moher et al., 2010; (Schulz, Altman, \& Moher, 2010).

Measures

Sociodemographic factors (such as age and gender), medical conditions, medications currently prescribed, past history of surgeries and medical procedures, smoking habits as well as alcohol consumption were obtained via a structured questionnaire. Anthropometry measures including body weight and height were measured using digital scales and a stadiometer respectively, and body mass index was calculated (weight $(\mathrm{kg}) /$ height $\left(\mathrm{m}^{2}\right)$ ).

Primary outcome: The Balance Outcome Measure for Elder Rehabilitation (BOOMER)

The BOOMER battery test was used as the primary outcome to assess the effectiveness of the novel purpose-built exercise park in improving balance. This test is a multi-item balance measure, which comprises four well validated clinical measures (step test (Hill, Bernhardt, McGann, Maltese, \& Berkovits, 1996), timed up and go (TUG) (Shumway-Cook, Brauer, \& Woollacott, 2000), functional reach (FRT) (Duncan, Weiner, Chandler, \& Studenski, 1990), and static standing balance (Anacker \& Di Fabio, 1992)) (Kuys, Morrison, Bew, Clarke, \& Haines, 2011).

Secondary Measures - Strength and Physical Function

The following secondary measures were used to assess balance, muscle strength, mobility and physical function in older adults. The single leg stance test standing on the dominant leg with eyes open was used to measure static balance (Springer, Marin, Cyhan, Roberts, \& Gill, 2007). The average hand grip strength of both hands using a TTM digital hand dynamometer 
(Mentone Educational Centre, Melbourne, VIC) (Tromp et al., 2001) was taken to measure physical strength. The two-minute walk test was used to assess exercise tolerance (Butland, Pang, Gross, Woodcock, \& Geddes, 1982) and functional mobility (Gijbels, Eijnde, \& Feys, 2011).

Lower limb strength was assessed via the 30-second sit-to-stand test (Csuka \& McCarty, 1985) and measurement of the strength of the knee extensor muscles using a purposely built force transducer (Lord, Menz, \& Tiedemann, 2003). Finally, the assessment of gait speed was performed with the use of the GaitRite ${ }^{\circledR}$ system (CIR System, Inc, Harverton PA) instrumented walkway system.

Secondary Measures - Feasibility

Feasibility was defined as the number of participants recruited and retained over the recruitment period, overall adherence and seasonal adherence, safety and adverse effects. Overall adherence to the exercise program was defined by the number of sessions attended: $100 \%$ adherence if an EPIG participant attended 35 sessions. EPIG participants' participation and attendance was recorded via a spreadsheet diary and was collected respectively by the exercise supervisor of that participant on each specific session or by the principal researcher.

Seasonal adherence was recorded as adherence over Summer (December to end of February), Fall (March to end of May), Winter (June to end of August) and Spring (September to end of November). Also, the number of sessions that were cancelled due to rainy, windy and excessively hot days (above $37^{\circ} \mathrm{C}$ ) were recorded given that these conditions potentially put participant's safety and health at risk. Safety and adverse effects were measured by the number of falls incidents during exercise sessions and muscle/joint injuries or strains reported after undertaking the exercise session. 
Secondary Outcomes - Health Related Quality of Life and Psychological Measures

The Short Form (12) Health Survey Version 2 (SF-12v2 ${ }^{\mathrm{TM}}$ ) was used to evaluate the individual health status (Ware Jr, Kosinski, \& Keller, 1996). Physical and Mental Health Composite Scores (PCS \& MCS) were computed using the scores of twelve questions and range from 0 to 100 , where a zero score indicates the lowest level of health measured by the scales and 100 indicates the highest level of health (Ware Jr et al., 1996).

The Short Falls Efficacy Scale International (Short FES-I) questionnaire was used to record fear of falling (Kempen et al., 2008). The total score ranges from 7 (not concerned) to 28 (severe concern) where higher scores are associated to a greater fear of falling (Kempen et al., 2008).

Secondary Measures - Physical activity and number of falls over 12 months

Physical activity calendars were used to monitor if EPIG or CG participants have participated in any other physical activities during their participation in the study and to monitor if the participants had falls over the 12 months from commencing involvement in the project. Calendars were returned using pre-paid envelopes and participants were followed up with a phone call in the cases their calendars were not returned within two weeks of the end of each month.

\section{Data Management and Statistical Analysis}

Power analysis was undertaken using previously published discharge data on the primary outcome measure - the BOOMER measure (Kuys et al., 2011), and assuming an improvement of 3 points (reported as the minimum detectable difference (Kuys et al., 2011)) and an effect size of 0.5. A sample size of 48 participants per group would be required for a power of 0.80 and alpha 
of 0.05 . A $20 \%$ dropout rate is anticipated based on previous exercise programs with older people. Therefore, this study aimed to recruit 60 participants per group.

All analyses were completed using SPSS version 22.0 and a p value less than 0.05 was considered statistically significant. Effect size $\left(\eta_{\mathrm{p}}{ }^{2}\right)$ from SPSS was used to determine effect size as follows: $\eta_{\mathrm{p}}{ }^{2}$ value greater than 0.14 was considered a large and significant effect size whereas 0.01 and 0.06 were considered small and medium, respectively (Cohen, 1988). For the primary outcome, the BOOMER test, repeated measures ANCOVA univariate analysis was performed to examine the difference between groups (baseline vs 18-week assessment) and between groups over time (group by time interaction) whilst controlling for age. Repeated measures ANCOVA were performed to evaluate the differences between groups (baseline vs 18-week assessment) and between groups over time on the individual components of BOOMER test, on the secondary physiological and quality of life and psychological outcomes. Ninety-five percent Confidence Interval $(95 \% \mathrm{CI})$ was calculated for the differences between the two groups over time. Age was included as a covariate given the decline of many physiological functions which happen with increasing age (Whitbourne, 2012). Prior to the conduct of the main analysis, data were grouped based on group preference (preferred and non-preferred group) and analysed to identify any possible effect of group allocation preference on the outcomes.

A mixed modelling analysis and the associated intention-to-treat approach were not used in the present study due to the following reasons: (1) for some participants, only one data point was available beyond baseline due to missing data (drop out) and (2) a disproportionately high number of participant dropouts from the control group as a result of not being allocated to the exercise intervention group. Furthermore, there is no adequate recommendation for replacement of missing values greater than 20\% (Unnebrink \& Windeler, 2001) and imputation of values for 
the missing data in similar cases to this study (dropout $>10 \%$ ) is likely to produce biased estimates of treatment effect (Simons-Morton, Obarzanek, \& Cutler, 2006). Therefore, a "per protocol" approach has been used in the analysis of the data. By using per protocol analysis, the risk of having the treatment effect underestimated or overestimated due to missing data is reduced (Baron, Boutron, Giraudeau, \& Ravaud, 2005) which will allow a more accurate representation of the actual effectiveness of the present exercise intervention (Armijo-Olivo, Warren, \& Magee, 2009). Similarly, only the data of participants assigned to the exercise intervention who actually received, complied with, and completed the treatment have been used (Armijo-Olivo et al., 2009). As it has been previously showed that older adults need to attend exercise programs at least once weekly to achieve muscle strength gains and improve neuromuscular performance (Taaffe, Duret, Wheeler, \& Marcus, 1999), participants from EPIG who did not comply with the exercise intervention and failed to attend at least one session per week (i.e. total attendance less than 50\%) were excluded from the statistical analysis.

\section{Results}

Sixty-two older people (mean age of $71.4 \pm 6.7$ years; 44 females; 18 males) living in the community volunteered to be part in the study. Challenges with recruitment meant that this final sample of sixty-two older people was lower than planned. More than $60 \%$ of participants of both groups had a history of at least one fall in the last 12 months. The mean age of participants in both groups was 70.2 years \pm 8.2 and 75.1 years \pm 7.9 for CG and EPIG, respectively with the majority of participants being females (77\% and $64 \%$ in CG and EPIG, respectively). Participants' characteristics are provided in Table 2. No significant differences were observed at baseline between the two groups. Seventy percent of the total dropout from the control group was due to participants not being allocated to the EPIG, further details about dropout and 
exclusion are provided in Figure 1. Table 3 provides information about the primary and secondary outcome measures.

\section{Treatment/Group Preference}

Ninety-six percent $(n=26)$ and $43 \%$ of participants from the intervention and control group, respectively, received their group preference. The difference between pre and post values for all outcome measures (delta change) based on participant preference was calculated and compared. As only one participant from the intervention received their non-preferred group allocation, analysis was conducted only for the control group. Multivariate analysis showed no differences between preferred and non-preferred group allocation $(\mathrm{p}>0.05)$.

\section{The exercise program}

All participants allocated to EPIG program performed all exercises as per description on the study protocol (Sales et al., 2015) with minimal adjustment based on individual abilities. All participants were able to perform all exercises as per the study protocol. These exercises were paired up in exercise stations as shown on Table 1. Additionally, an average of 35 sessions were run for each group of participants with the objective of reaching 50 hours of cumulative exercise previously suggested to be effective in reduction of falls risks for older adults (Sherrington et al., 2008).

Primary outcome: BOOMER Test

No significant difference was found between the groups at baseline and after 18-week participation for the BOOMER test or the BOOMER individual components $(\mathrm{p}>0.05)$. No significant differences were found for the BOOMER test between the groups over time $(\mathrm{p}=0.46$, 95\% CI -.354 to .830) (Table 3). The component tests of BOOMER test were also analysed individually and no significant differences were observed between the groups over time $(\mathrm{p}>0.05)$. 
Secondary Outcomes - Strength and Physical Function

Participants from EPIG showed significant improvement on single leg stance $(\mathrm{p}=0.02$, 95\% CI -8.35 to -.549 ), knee strength ( $\mathrm{p}<0.01,-29.14$ to -5.86 ), two-minute walk ( $\mathrm{p}=0.02$, 19.13 to -.859$)$ and timed sit to stand ( $\mathrm{p}=0.03,-2.26$ to -.143$)$ tests following the 18 -week intervention compared to the CG (Table 3). A significant difference was found between the groups only for the hand grip strength $(\mathrm{p}=0.01$, Table 3$)$. Univariate t-test revealed greater strength for the EPIG at the follow up assessment $(\mathrm{p}=0.04,95 \% \mathrm{CI}-10.39$ to -0.11$)$.

Secondary Outcomes - Feasibility

Twenty-seven participants from EPIG completed the 18-week intervention (87\%) with mean attendance of $79.6 \%$. However, only $14 \%$ of participants in the control group attended to the social meetings offered. Summer and autumn were the seasons that demonstrated the highest levels of attendance with participants respectively attending $86.1 \%$ and $72.7 \%$ (Figure 3). Two falls during exercise sessions were reported with two participants. One participant missed the seat during the sit-to-stand exercise and the other lost balance when stepping down from a platform. No injuries or adverse events were reported from these two episodes nor during the research trial. Only $9.6 \%$ of sessions had to be cancelled due to rain. Also, no participants in the exercise intervention group reported experiencing uncomfortable delayed muscle soreness or fatigue post-exercise that limited them from doing their daily tasks.

Secondary Outcomes - Fear of Falling and Quality of Life

No significant differences were found for fear of falling (Short FES-I, $p=0.4,95 \% \mathrm{CI}$ 1.10 to 2.05 ) and quality of life (SF-12 PCS and MCS respectively, $\mathrm{p}=0.2,-7.24$ to $1.37 ; \mathrm{p}=$ 0.6, -5.76 to 3.22) between EPIG and CG over time.

Secondary Measures - Number of Falls Over 12 months 
No significant difference was found for the number of falls between EPIG and CG after 12 months $(\mathrm{p}=0.78)$; although there was a reduction of $35.2 \%$ in the number of fallers among EPIG participants (Table 2) and 23.1\% among CG participants.

\section{Discussion}

Participation in the 18-week exercise program using the purpose-built exercise park resulted in improvement in muscle strength, balance and physical function in older adults. Furthermore, given the relatively high attendance and retention rates observed among participants allocated to the exercise intervention group and the absence of major adverse events which could compromise the safety of participants, this novel concept might be a feasible option to improve participation and adherence to exercise programs aiming to reduce falls among older adults.

The importance of balance in preventing falls among older adults is well established (Howe, Rochester, Neil, Skelton, \& Ballinger, 2011). As balance is multi-dimensional, the BOOMER, a test battery that incorporates a number of key domains of balance (static and dynamic balance, including measures of stepping, reaching and turning, that are commonly involved in falls) was used in this present study. Our results showed no significant improvements in the BOOMER test among participants from the intervention group across time. Participants of both groups, at baseline, nearly reached the maximum BOOMER score value of 16 (CG mean $=13.4$, EPIG mean $=13.5$ ) and the same was observed when evaluating each component test individually suggesting that only minimal improvement can be achieved. Hence, the lack of improvement could be a reflection of a ceiling effect and that this chosen primary outcome measure may not be sensitive enough to be used on a sample of independent and mostly healthy 
community dwelling seniors. Previous research on the BOOMER has only used older adults in geriatric and rehabilitation units (Haines et al., 2007).

Exercise interventions targeting improvement in muscle strength, balance, mobility, agility, and functional tasks have been reported to be effective in reducing risk of falls and the number of falls among older adults (Karlsson, Vonschewelov, Karlsson, Cöster, \& Rosengen, 2013). In the present study, significant improvements in knee strength, balance (single leg stance), two-minute walk test and sit to stand were demonstrated following the eighteen-week exercise intervention with light to moderate but still challenging exercises. A non-significant reduction in the number of falls by $35.2 \%$ for the exercise intervention group was also reported, although this sample is underpowered for identifying a significant difference on this outcome. The ability to walk and function are important to reduce disability and promote independent living among older adults (Seidel, Brayne, \& Jagger, 2011). Similarly, muscle strength and balance have been reported to be critical elements responsible for maintaining physical function, mobility and vitality in old age (Haines et al., 2007). Given the prescribed exercises were similar to daily tasks required in daily life, the functional and translational aspect of the exercises proposed might have contributed to the positive outcome on the physical and mobility measures which can positively affect confidence and self-efficacy (Lee, Arthur, \& Avis, 2008). Interventions which particularly target exercise self-efficacy, perceived exercise enjoyment, confidence and satisfaction are more prone to promote behavioural change in older adults (Lee et al., 2008). A further investigation with a larger sample size is now needed to evaluate if using the senior exercise park would also result in reduction in the number of falls among older adults.

Despite the improvements in physical performance and function, no improvements in quality of life and falls efficacy were found in the present study. Participants from both groups, 
were not only more independent and physically capable but also presented with relatively high baseline values for the quality of life measures compared to the reported Australian population aged 70+ (Table 3) (Tucker, Adams, \& Wilson, 2010). Similarly, although presenting some concern about the possibility of having a fall or a history of fall, current participants of this study showed relatively low fear of falling (Table 3) when compared to the average value expected for older adults between 70-80 years (Kempen et al., 2008). Therefore, older people with lower levels of quality of life and with greater fear of falling might result in greater improvement in these domains when accessing the senior exercise park intervention, however this would require further investigation.

Although exercise has been shown to be an important and effective approach to preventing falls in older people (Sherrington et al., 2008), adherence to exercise programs remains a persistent problem (Chao, Foy, \& Farmer, 2000). In this present study, a high adherence rate of $87 \%$ and attendance rate of $79.6 \%$ was reported among participants in the exercise intervention group. The social context and support element of the EPIG program, which participants called "the get-together moment", provided the sessions with a playful and relaxed atmosphere that probably camouflaged the physical challenge they were facing while exercising throughout different stations. Furthermore, offering physical sessions with high emphasis on social support (e.g., social support provided by exercise classmates and exercise team leader) is a key way to achieve success with the adherence and retention of older adults participating in exercise interventions (Brassington, Atienza, Perczek, DiLorenzo, \& King, 2002). The high attendance and adherence observed might also be related to the sessions being run outdoors despite the unpredictability of Melbourne's weather. Importantly, it has been shown that older 
adults tend to favour attending outdoor sessions over the indoor sessions (Barton et al., 2012) and that outdoor exercises greatly improve mood in older people (Barton et al., 2012).

Apart from the two minor fall episodes which happened during the exercise session delivery, no adverse events nor muscle strains or injuries that needed further medical intervention were reported during the trial. However, it is important to note that participants were working in pairs and had one exercise physiologist closely accompanying and supervising them throughout the entire session. To allow for broader public use of the exercise park, a simplification of the exercise protocol might be required so that older participants would be able to exercise more independently, but safely. Additionally, older adults could come and meet an allied health professional at a pre-established frequency (e.g. once a month) to proceed with establishing and progression of their exercise routine.

\section{Limitations}

While this study provides useful information about the effectiveness of an exercise intervention using an outdoor senior exercise park, several limitations are acknowledged. Firstly, we had an overall relatively modest sample size. The BOOMER test battery chosen as the primary outcome was not adequately sensitive to the population group studied. Furthermore, it is believed that the involvement, adherence and attendance to this project could have been higher if the senior exercise park had been installed in a location accessible by public transport and in a more central suburb of Melbourne. For future trials, location of the exercise park needs to be considered to allow easy access. Moreover, to facilitate the attrition of participants in a control group and to minimise dropout rate (as observed in this study), the control group should be offered some other non-physical activities which are perceived as meaningful for older people in combination with social activities rather than solely social activities. 


\section{Conclusion}

The 18-week exercise program using a senior exercise park has been shown to be effective and safe in improving balance, muscle strength and physical function among older adults, and, therefore, may reduce the risk of falling in older people living in the community. This initiative demonstrated high adherence and participation rate. However, further investigation with a larger sample size is now needed to evaluate if the exercise park intervention would also be effective in reducing the number of falls among older adults.

\section{Abbreviations}

Short-FES-I: The Short Falls Efficacy Scale International; SF12-PCS and SF12-MCS: Physical and Mental Component scores of the Short Form (12) Health Survey (SF-12); EPIG: Exercise Park Intervention Group; CG: Control Group; BOOMER: Balance Outcome Measure for Elder Rehabilitation.

\section{Conflict of interests}

The authors declare that they have no conflict of interests. The authors declare no support from any organisation for the submitted work; no financial relationships with any organisations that might have an interest in the submitted work in the previous three years, no other relationships or activities that could appear to have influenced the submitted work. 


\section{References}

Anacker, S. L., \& Di Fabio, R. P. (1992). Influence of sensory inputs on standing balance in communitydwelling elders with a recent history of falling. Physical Therapy, 72(8), 575-581.

Armijo-Olivo, S., Warren, S., \& Magee, D. (2009). Intention to treat analysis, compliance, drop-outs and how to deal with missing data in clinical research: a review. Physical Therapy Reviews, 14(1), 3649.

Baron, G., Boutron, I., Giraudeau, B., \& Ravaud, P. (2005). Violation of the intent-to-treat principle and rate of missing data in superiority trials assessing structural outcomes in rheumatic diseases. Arthritis and Rheumatism, 52(6), 1858-1865.

Barton, J., Griffin, M., \& Pretty, J. (2012). Exercise-, nature-and socially interactive-based initiatives improve mood and self-esteem in the clinical population. Perspectives in public health, 132(2), 8996.

Brassington, G. S., Atienza, A. A., Perczek, R. E., DiLorenzo, T. M., \& King, A. C. (2002). Interventionrelated cognitive versus social mediators of exercise adherence in the elderly. American Journal of Preventive Medicine, 23(2), 80-86.

Butland, R. J., Pang, J., Gross, E. R., Woodcock, A. A., \& Geddes, D. M. (1982). Two-, six-, and 12minute walking tests in respiratory disease. British Medical Journal, 284(6329), 1607-1608.

Chao, D., Foy, C. G., \& Farmer, D. (2000). Exercise adherence among older adults: challenges and strategies. Controlled Clinical Trials, 21(5), S212-S217.

Cohen, J. (1988). Statistical power analysis for the behavioral sciences (2 ed.). Hillside, NJ: Lawrence Erlbaum Associates.

Csuka, M., \& McCarty, D. J. (1985). Simple method for measurement of lower extremity muscle strength. The American Journal of Medicine, 78(1), 77-81.

Duncan, P. W., Weiner, D. K., Chandler, J., \& Studenski, S. (1990). Functional reach: a new clinical measure of balance. The Journals of Gerontology, 45(6), M192-197.

Evron, L., Schultz-Larsen, K., \& Fristrup, T. (2009). Barriers to participation in a hospital-based falls assessment clinic programme: an interview study with older people. Scandinavian journal of public health.

Gijbels, D., Eijnde, B., \& Feys, P. (2011). Comparison of the 2-and 6-minute walk test in multiple sclerosis. Multiple Sclerosis Journal, 17(10), 1269-1272.

Granacher, U., Muehlbauer, T., Gollhofer, A., Kressig, R. W., \& Zahner, L. (2011). An intergenerational approach in the promotion of balance and strength for fall prevention-a mini-review. Gerontology, 57(4), 304-315.

Haines, T., Kuys, S. S., Morrison, G., Clarke, J., Bew, P., \& McPhail, S. (2007). Development and validation of the balance outcome measure for elder rehabilitation. Archives of Physical Medicine and Rehabilitation, 88(12), 1614-1621. doi:10.1016/j.apmr.2007.09.012

Hill, K., Bernhardt, J., McGann, A., Maltese, D., \& Berkovits, D. (1996). A new test of dynamic standing balance for stroke patients: reliability, validity and comparison with health elderly. Physiotherapy Canada, 48, 257-262.

Howe, T., Rochester, L., Neil, F., Skelton, D., \& Ballinger, C. (2011). Exercise for improving balance in older people (Review). Cochrane Database Syst Rev, 9(11), CD004963.

Karlsson, M. K., Vonschewelov, T., Karlsson, C., Cöster, M., \& Rosengen, B. E. (2013). Prevention of falls in the elderly: a review. Scandinavian journal of public health, 41(5), 442-454.

Kempen, G. I., Yardley, L., van Haastregt, J. C., Zijlstra, G. A., Beyer, N., Hauer, K., \& Todd, C. (2008). The Short FES-I: a shortened version of the falls efficacy scale-international to assess fear of falling. Age and Ageing, 37(1), 45-50.

Kuys, S. S., Morrison, G., Bew, P. G., Clarke, J., \& Haines, T. P. (2011). Further validation of the Balance Outcome Measure for Elder Rehabilitation. Archives of Physical Medicine and Rehabilitation, 92(1), 101-105. doi:10.1016/j.apmr.2010.10.001

Lee, L.-L., Arthur, A., \& Avis, M. (2008). Using self-efficacy theory to develop interventions that help older people overcome psychological barriers to physical activity: a discussion paper. International Journal of Nursing Studies, 45(11), 1690-1699. 
Lord, S. R., Menz, H. B., \& Tiedemann, A. (2003). A physiological profile approach to falls risk assessment and prevention. Physical Therapy, 83(3), 237-252.

McMahon, S., Talley, K. M., \& Wyman, J. F. (2011). Older people's perspectives on fall risk and fall prevention programs: a literature review. International Journal of Older People Nursing, 6(4), 289298. doi:10.1111/j.1748-3743.2011.00299.x

Moher, D., Hopewell, S., Schulz, K. F., Montori, V., Gøtzsche, P. C., Devereaux, P., . . Altman, D. G. (2010). CONSORT 2010 explanation and elaboration: updated guidelines for reporting parallel group randomised trials. Journal of Clinical Epidemiology, 63(8), e1-e37.

Paterson, D. H., Jones, G. R., \& Rice, C. L. (2007). Ageing and physical activity: evidence to develop exercise recommendations for older adults. Applied Physiology, Nutrition, and Metabolism, 32(S2E), S69-S108.

Sales, M. P., Polman, R., Hill, K. D., Karaharju-Huisman, T., \& Levinger, P. (2015). A novel dynamic exercise initiative for older people to improve health and well-being: study protocol for a randomised controlled trial. BMC Geriatrics, 15(1), 68.

Schulz, K. F., Altman, D. G., \& Moher, D. (2010). CONSORT 2010 statement: updated guidelines for reporting parallel group randomised trials. BMC Medicine, 8(1), 18.

Seidel, D., Brayne, C., \& Jagger, C. (2011). Limitations in physical functioning among older people as a predictor of subsequent disability in instrumental activities of daily living. Age and Ageing, afr054.

Sherrington, C., Whitney, J. C., Lord, S. R., Herbert, R. D., Cumming, R. G., \& Close, J. C. (2008). Effective exercise for the prevention of falls: a systematic review and meta-analysis. Journal of the American Geriatrics Society, 56(12), 2234-2243.

Shumway-Cook, A., Brauer, S., \& Woollacott, M. (2000). Predicting the probability for falls in communitydwelling older adults using the Timed Up \& Go Test. Physical Therapy, 80(9), 896-903.

Simons-Morton, D. G., Obarzanek, E., \& Cutler, J. A. (2006). Obesity research-limitations of methods, measurements, and medications. JAMA, 295(7), 826-828.

Springer, B. A., Marin, R., Cyhan, T., Roberts, H., \& Gill, N. W. (2007). Normative values for the unipedal stance test with eyes open and closed. Journal of Geriatric Physical Therapy, 30(1), 8-15.

Taaffe, D. R., Duret, C., Wheeler, S., \& Marcus, R. (1999). Once-weekly resistance exercise improves muscle strength and neuromuscular performance in older adults. Journal of the American Geriatrics Society, 47(10), 1208-1214.

Tromp, A., Pluijm, S., Smit, J., Deeg, D., Bouter, L., \& Lips, P. (2001). Fall-risk screening test: a prospective study on predictors for falls in community-dwelling elderly. Journal of Clinical Epidemiology, 54(8), 837-844.

Tucker, G., Adams, R., \& Wilson, D. (2010). New Australian population scoring coefficients for the old version of the SF-36 and SF-12 health status questionnaires. Quality of Life Research, 19(7), 1069-1076.

Unnebrink, K., \& Windeler, J. (2001). Intention-to-treat: methods for dealing with missing values in clinical trials of progressively deteriorating diseases. Statistics in Medicine, 20(24), 3931-3946.

Van Kan, G. A., Rolland, Y., Andrieu, S., Bauer, J., Beauchet, O., Bonnefoy, M., . . Inzitari, M. (2009). Gait speed at usual pace as a predictor of adverse outcomes in community-dwelling older people an International Academy on Nutrition and Aging (IANA) Task Force. The Journal of Nutrition, Health \& Aging, 13(10), 881-889.

Ware Jr, J. E., Kosinski, M., \& Keller, S. D. (1996). A 12-Item Short-Form Health Survey: construction of scales and preliminary tests of reliability and validity. Medical Care, 34(3), 220-233.

Whitbourne, S. K. (2012). The aging body: Physiological changes and psychological consequences: Springer Science \& Business Media.

Yardley, L., Donovan-Hall, M., Francis, K., \& Todd, C. (2006). Older people's views of advice about falls prevention: a qualitative study. Health Education Research, 21(4), 508-517.

Yardley, L., Kirby, S., Ben-Shlomo, Y., Gilbert, R., Whitehead, S., \& Todd, C. (2008). How likely are older people to take up different falls prevention activities? Preventive Medicine, 47(5), 554-558. 
Table 1: Exercise stations.

\begin{tabular}{lll}
\hline $\begin{array}{l}\text { Station } \\
\text { Number }\end{array}$ & Exercise 1 & Exercise 2 \\
\hline 1 & & \\
\hline 2 & Push-ups & Taps on Platform \\
3 & Modified Pull-ups & Gangway \\
& Balance Stool & Calf Raises + Finger \\
4 & & Steps \\
5 & Sit to Stand & Round Snake Pipe \\
& Ramp + Net + Climb & Sharp Snake Pipe \\
6 & Through & \\
7 & Balance Beam & Hip extension \\
8 & Steps & Screws / Turners \\
\hline
\end{tabular}


Table 2: General characteristics of the participants of this study

\begin{tabular}{lcc}
\hline Characteristic & $\begin{array}{c}\text { Control Group } \\
(\mathbf{n = 2 1})\end{array}$ & $\begin{array}{c}\text { Exercise } \\
\text { Intervention Group } \\
(\mathbf{n = 2 7})\end{array}$ \\
\hline Age (Years) & $70.2 \pm 8.20$ & $75.1 \pm 7.95$ \\
Gender (Females, \%) & 77 & 64 \\
BMI (kg/m $\left.{ }^{2}\right)$ & $28.1 \pm 5.04$ & $28.9 \pm 5.34$ \\
Current Smoker (\%) & 6 & 3 \\
Ex-Smoker (\%) & 29 & 42 \\
Daily Alcohol Consumption (\%) & 52 & 3 \\
Average Number of Medications & 3 & 62.9 \\
Previous Falls History (>=1fall, \%) & 61.9 & 40.7 \\
Follow-up Falls Over 12 months (\%) & 47.6 & 31 \\
\hline
\end{tabular}


Table 3: Primary and secondary outcome measures before and after the 18-week participation for the CG and EPIG (values are mean \pm $\mathrm{SD})$

\begin{tabular}{|c|c|c|c|c|c|c|c|}
\hline \multirow{2}{*}{ Measure } & \multicolumn{2}{|c|}{$\begin{array}{c}C G \\
(n=21)\end{array}$} & \multicolumn{2}{|c|}{$\begin{array}{c}E P I G \\
(n=27)\end{array}$} & \multirow{2}{*}{$\begin{array}{l}\text { P value Group by Time } \\
\text { Interaction }(95 \% \text { CI })\end{array}$} & \multirow{2}{*}{$\eta_{p}^{2}$} & \multirow{2}{*}{$\begin{array}{c}\text { p value } \\
\text { between } \\
\text { groups }\end{array}$} \\
\hline & Pre & Post & Pre & Post & & & \\
\hline BOOMER - Total Score (Out of 16) & $13.5 \pm 1.7$ & $13.9 \pm 1.4$ & $13.6 \pm 1.4$ & $13.7 \pm 1.3$ & $0.6(-.354$ to .830$)$ & 0.00 & 0.4 \\
\hline \multicolumn{8}{|l|}{ Physical Measures } \\
\hline Single Leg Stance (sec) & $18.7 \pm 11.1$ & $16.0 \pm 9.2$ & $15.6 \pm 11.0$ & $17.3 \pm 11.3$ & $\mathrm{p}<0.01 *(-8.35$ to -.549$)$ & $0.11^{\ddagger}$ & 0.4 \\
\hline Knee Strength (N.m) & $83.7 \pm 33.1$ & $78.5 \pm 33.8$ & $84.2 \pm 36.5$ & $96.4 \pm 44.4$ & $\mathrm{p}<0.01 *(-29.1$ to -5.86$)$ & $0.15^{\phi}$ & 0.1 \\
\hline Hand Grip Strength (Kg) & $20.6 \pm 7.2$ & $20.9 \pm 7.1$ & $26.3 \pm 10.6$ & $26.5 \pm 9.6$ & $0.4(-1.52$ to 1.88$)$ & $0.02 \dagger$ & $0.01 * *$ \\
\hline Two Minute Walk (m) & $149.0 \pm 29.5$ & $150.4 \pm 22.5$ & $140.6 \pm 30.5$ & $152.1 \pm 28.7$ & $\mathrm{p}<0.01 *(-19.1$ to -.859$)$ & $0.12^{\ddagger}$ & 0.7 \\
\hline Timed Up and Go Fast (sec) & $7.0 \pm 2.0$ & $7.0 \pm 1.4$ & $7.4 \pm 1.8$ & $7.1 \pm 1.4$ & $0.6(-.315$ to .913$)$ & $0.01 \dagger$ & 0.8 \\
\hline Sit to Stand (reps) & $11.0 \pm 2.2$ & $11.5 \pm 2.5$ & $10.5 \pm 3.0$ & $12.1 \pm 2.7$ & $\mathrm{p}<0.01 *(-2.26$ to -.143$)$ & $0.10^{\ddagger}$ & 0.6 \\
\hline Gait Speed (m/s) & $1.34 \pm 20.2$ & $1.36 .5 \pm 16.0$ & $1.31 \pm 19.2$ & $1.33 \pm 17.0$ & $0.8(-6.11$ to 4.30$)$ & 0.00 & 0.8 \\
\hline \multicolumn{8}{|l|}{ Fear of Falling and Quality of Life } \\
\hline Short FES-I & $11.3 \pm 4.0$ & $10.9 \pm 3.7$ & $10.3 \pm 3.4$ & $9.3 \pm 2.5$ & $0.4(-1.10$ to 2.05$)$ & $0.02 \dagger$ & 0.1 \\
\hline SF12 PCS & $49.1 \pm 7.91$ & $48.9 \pm 7.6$ & $46.9 \pm 7.56$ & $49.6 \pm 8.29$ & $0.2(-7.24$ to 1.37$)$ & $0.03 \dagger$ & 0.8 \\
\hline SF12 MCS & $51.4 \pm 6.1$ & $51.6 \pm 7.9$ & $53.1 \pm 9.8$ & $54.5 \pm 7.0$ & $0.6(-5.76$ to 3.22$)$ & 0.01 & 0.4 \\
\hline
\end{tabular}


24| P a g e 


\section{CONSORT Flow Diagram of Recruitment and Randomization}

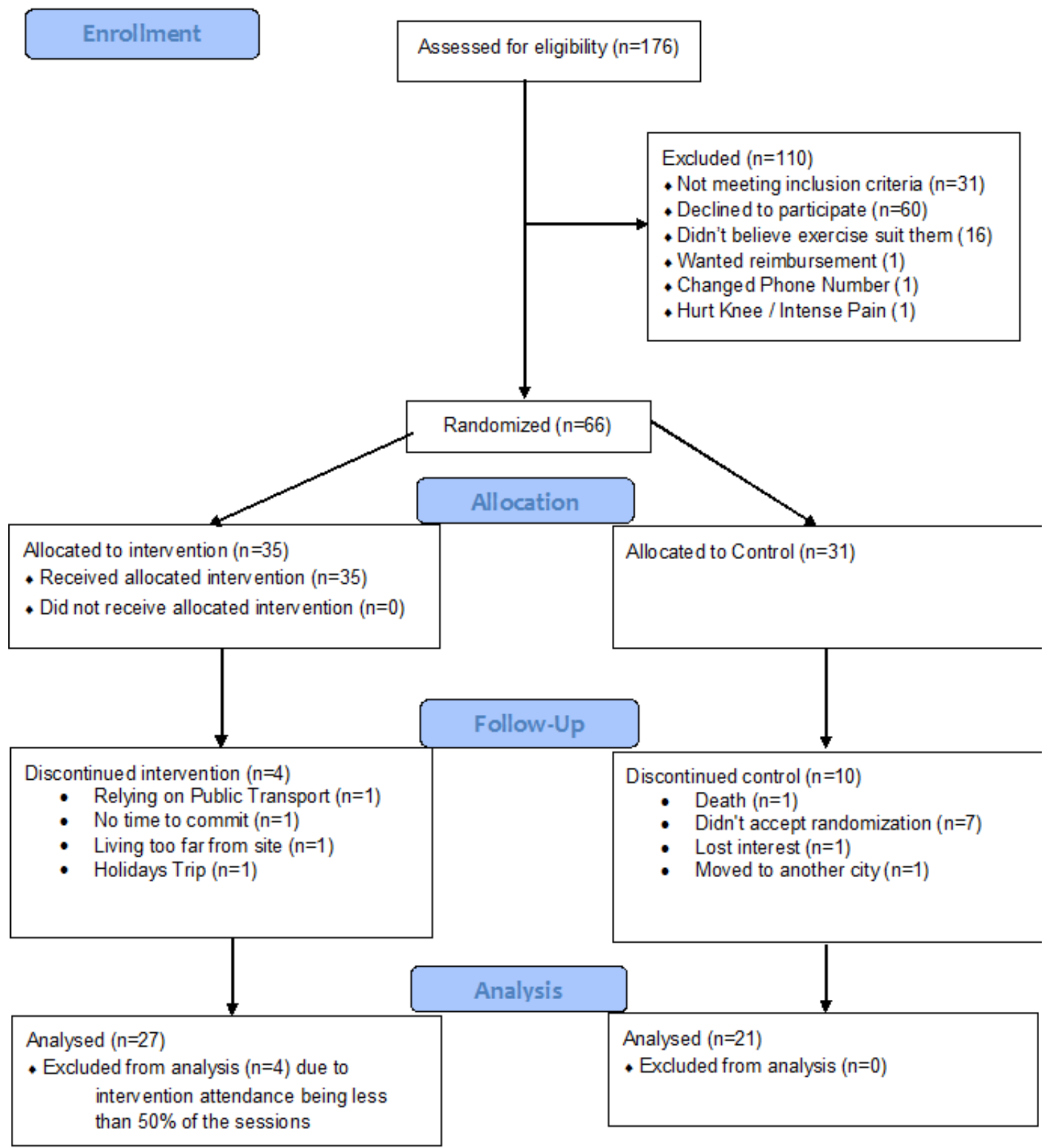

Figure 1: Consort flow diagram of recruitment and randomization. 


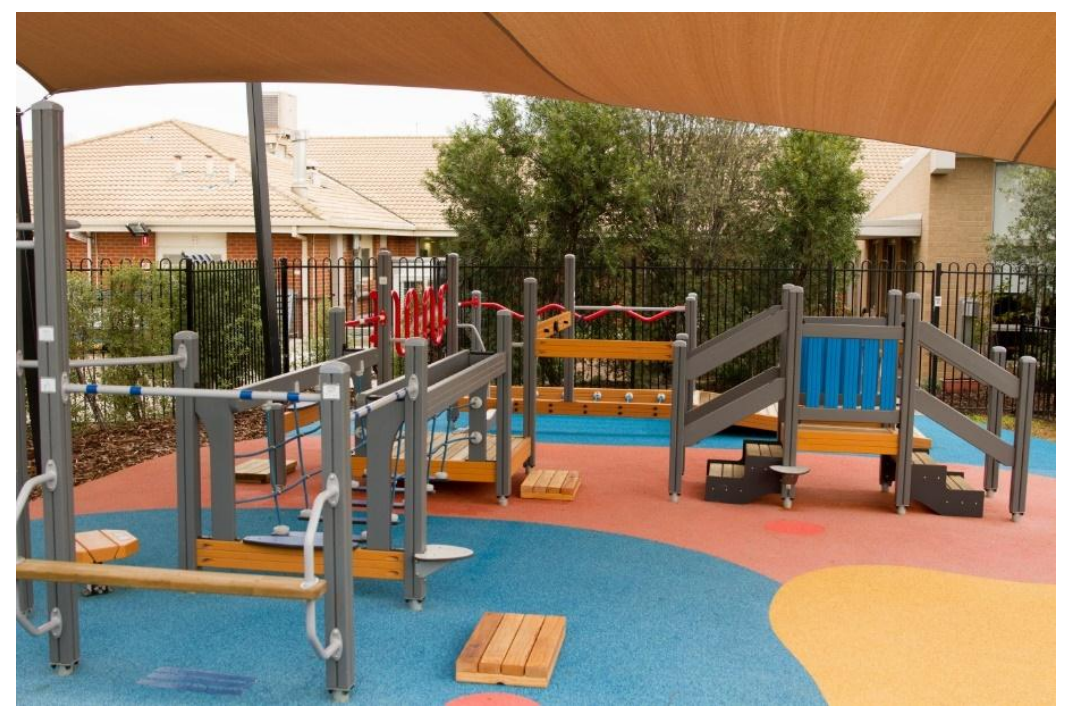

Figure 2: Lappset's exercise park for senior population.

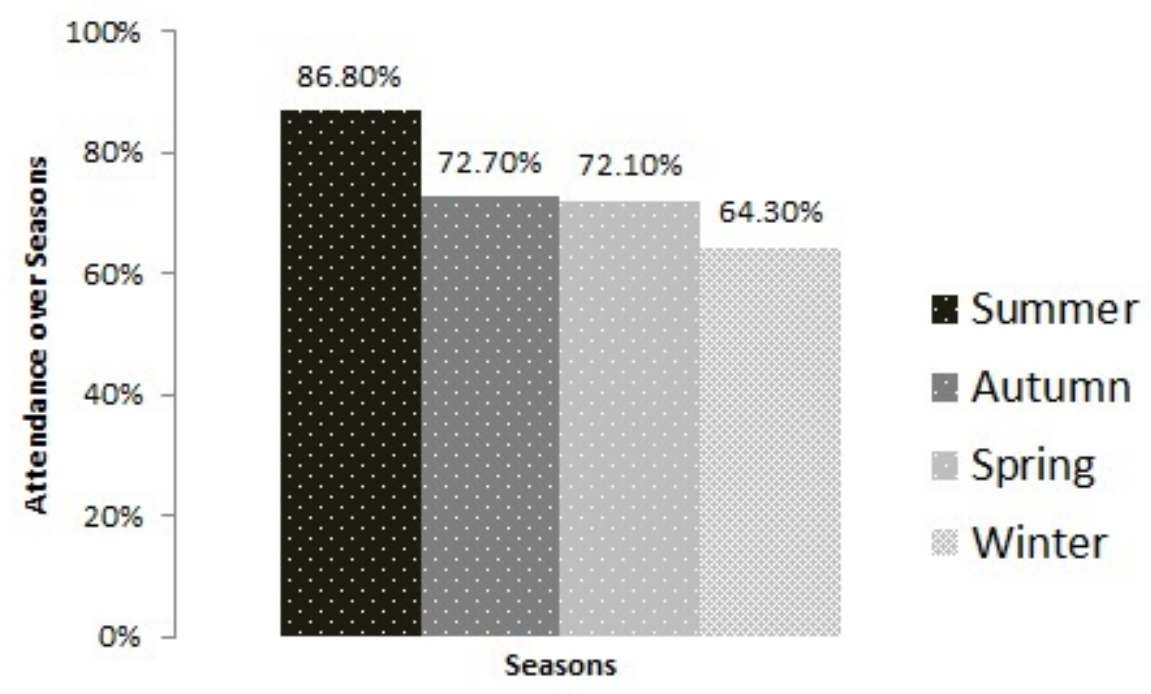

Figure 3: Participant's attendance throughout the different seasons 